\title{
Exemplar scoring identifies genetically separable phenotypes of lithium responsive bipolar disorder
}

\author{
Abraham Nunes $\mathbb{D}^{1,2}$, William Stone ${ }^{2}$, Raffaella Ardau ${ }^{3}$, Anne Berghöfer $\mathbb{D}^{4}$, Alberto Bocchetta ${ }^{3}$, Caterina Chillotti ${ }^{3}$, \\ Valeria Deiana ${ }^{5}$, Franziska Degenhardt ${ }^{6}$, Andreas J. Forstner $0^{6,7,8}$, Julie S. Garnham (1) ${ }^{1}$ Eva Grof ${ }^{9}, 10$, Tomas Hajek (D)', \\ Mirko Manchia (10 ${ }^{11,12}$, Manuel Mattheisen ${ }^{13}$, Francis McMahon (1) ${ }^{14}$, Bruno Müller-Oerlinghausen ${ }^{15}$, Markus M. Nöthen ${ }^{6}$,

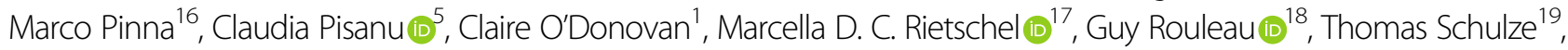 \\ Giovanni Severino $\mathbb{0}^{5}$, Claire M. Slaney ${ }^{1}$, Alessio Squassina (10), Aleksandra Suwalska (10 ${ }^{20,21}$, Gustavo Turecki (i) ${ }^{22}$, \\ Rudolf Uher ${ }^{1}$, Petr Zvolsky ${ }^{23}$, Pablo Cervantes ${ }^{22}$, Maria del Zompo ${ }^{5}$, Paul Grof ${ }^{9,10}$, Janusz Rybakowski ${ }^{20,24}$, \\ Leonardo Tondo ${ }^{16,25}$, Thomas Trappenberg ${ }^{2}$ and Martin Alda (i) ${ }^{1}$
}

\begin{abstract}
Predicting lithium response (LiR) in bipolar disorder (BD) may inform treatment planning, but phenotypic heterogeneity complicates discovery of genomic markers. We hypothesized that patients with "exemplary phenotypes" - those whose clinical features are reliably associated with LiR and non-response (LiNR) - are more genetically separable than those with less exemplary phenotypes. Using clinical data collected from people with BD ( $n=1266$ across 7 centers; $34.7 \%$ responders), we computed a "clinical exemplar score," which measures the degree to which a subject's clinical phenotype is reliably predictive of LiR/LiNR. For patients whose genotypes were available $(n=321)$, we evaluated whether a subgroup of responders/non-responders with the top $25 \%$ of clinical exemplar scores (the "best clinical exemplars") were more accurately classified based on genetic data, compared to a subgroup with the lowest $25 \%$ of clinical exemplar scores (the "poor clinical exemplars"). On average, the best clinical exemplars of LiR had a later illness onset, completely episodic clinical course, absence of rapid cycling and psychosis, and few psychiatric comorbidities. The best clinical exemplars of LiR and LiNR were genetically separable with an area under the receiver operating characteristic curve of 0.88 (IQR $[0.83,0.98])$, compared to $0.66[0.61,0.80](p=0.0032)$ among poor clinical exemplars. Variants in the Alzheimer's amyloid-secretase pathway, along with G-protein-coupled receptor, muscarinic acetylcholine, and histamine H1R signaling pathways were informative predictors. This study must be replicated on larger samples and extended to predict response to other mood stabilizers.
\end{abstract}

\section{Introduction}

Bipolar disorder (BD) is a lifelong illness characterized by recurrent manias, depressions, and a relatively high suicide risk $^{1,2}$. Initiation of mood stabilizers, of which lithium is a first-line option ${ }^{3}$, occurs approximately a decade after symptom onset on average ${ }^{4}$, and the trial-and-error

\footnotetext{
Correspondence: Martin Alda (malda@dal.ca)

${ }^{1}$ Department of Psychiatry, Dalhousie University, Halifax, NS, Canada

${ }^{2}$ Faculty of Computer Science, Dalhousie University, Halifax, NS, Canada

Full list of author information is available at the end of the article

Deceased Petr Zvolsky
}

process of pharmacological optimization for BD may lengthen this time. However, by predicting patients' moodstabilizer response, this burden of untreated illness may be reduced.

Clinical phenotypes---patient features that may be obtained by clinical interview alone---are currently the best predictors of lithium response ${ }^{5}$, but genomic markers likely exist. Responders often have a "classical phenotype" characterized by a completely episodic course with full inter-episode remissions, absence of rapid cycling and psychosis (particularly if mood-incongruent), and family 
history of fully remitting $\mathrm{BD}$ or lithium response in a first degree relative ${ }^{6,7}$. The familial nature of lithium responsive BD has been a particular motivator for the pursuit of strong genomic markers of lithium response, and polygenic scores for major depression and schizophrenia are inversely associated with lithium responsiveness ${ }^{8,9}$. However, predictors based on variation measured by single nucleotide polymorphisms (SNPs) remain elusive ${ }^{10}$.

The significant clinical heterogeneity of BD may limit the power of genomic prediction of lithium response ${ }^{10}$. Indeed, we have found that the clinical predictors of lithium responsiveness may differ across centers in multisite studies ${ }^{5}$. However, some clinical phenotypes may consistently predict lithium responsiveness across all centers; patients with such phenotypes are the best clinical exemplars of lithium response and non-response, respectively. Conversely, poor clinical exemplars are characterized by clinical profiles that do not consistently predict lithium response or non-response. We hypothesize that biological differences will be greatest among samples of lithium responders and non-responders whose phenotypes are clinically exemplary.

The present work has two steps. First, by using a large set of clinical data on lithium-treated patients with $\mathrm{BD}$ collected across seven international specialist clinics, our present work develops a measure called clinical exemplar scoring which identifies those subjects whose lithium responsiveness can be reliably predicted from their clinical phenotype (the "best clinical exemplars" of lithium response and non-response, respectively). This method also identifies "poor clinical exemplars" of lithium response and non-response, respectively. Poor clinical exemplars are patients whose lithium responsiveness is not reliably predicted from their clinical profiles. We hypothesized that the clinical differences between the best clinical exemplars of lithium response and non-response would be reflective of factors previously associated with the "classical" bipolar phenotype.

The second step of our study leveraged the fact that genomic data were available for some subjects whose clinical exemplar scores were computed based on clinical features. The genomic and clinical data are not combined, but rather, we test whether genomic classification of responders vs. non-responders improves when the subject sample is restricted to only the best clinical exemplars of lithium response and non-response, respectively. We hypothesized that lithium responsiveness would be better discriminated among the best clinical exemplars, rather than the poor clinical exemplars.

\section{Methods}

Our analysis has two steps, using two separate datasets. Step 1 uses a multi-center database of clinical variables to derive a score that identifies subjects whose clinical phenotypes reliably predict lithium response/nonresponse. A subset of subjects for whom we computed clinical exemplar scores in Step 1 (a North American outbred sample from Dalhousie University, Canada) also had genomic data available from participation in an entirely separate study through the Consortium on Lithium Genetics (ConLiGen). In Step 2, we evaluate whether lithium responsiveness can be better discriminated based on genomic data when the subject group is restricted only to those individuals who are the best clinical exemplars of the lithium responsive and nonresponsive phenotypes, respectively. It bears repeating that absolutely no genomic data from Step 2 were included in the clinical exemplar scoring procedure of Step 1, and no clinical variables from Step 1 were included in the genomic classification procedure of Step 2. Clinical and genetic data were collected with informed consent in the context of protocols approved by the Ethics Committee of the former Health Agency of Cagliari (for Cagliari University and Centro Bini samples), and the research ethics boards of the Nova Scotia Health Authority, the McGill University Health Centre, the Royal Ottawa Hospital, and the University of Poznan.

\section{Step 1: Scoring and characterization of clinical exemplars}

Figure 1 illustrates Step 1 of the present study, wherein we identify and characterize clinical phenotypes that are reliably predictive of lithium responsiveness using the clinical exemplar score. This step of our study uses only the clinical database, without any reference to the genomic data used for Step 2 of the analysis.

\section{Clinical data collection}

Clinical data collection procedures were described in Nunes et al. ${ }^{5}$. Data consisted of 180 variables recorded prior to instituting lithium maintenance therapy in 1266 people with BD across 7 centers (minimum treatment duration of 1 year). Datasets are described in Supplementary Table 1. Lithium response was defined as a score of $\geq 7$ on the previously validated Alda scale ${ }^{11}$, which accounts for observation of appropriate lithium levels and compliance.

\section{Computation of the clinical exemplar score}

The best clinical exemplars should be classified accurately by models trained on data from any given site. Our overall clinical exemplar scoring protocol thus involves (A) obtaining out-of-sample predictions of every subject's class based on models trained on each individual site's data, then (B) summarizing accuracy and between-site agreement into a single value known as the clinical exemplar score. Figure 1 provides a visual intuition for the clinical exemplar score computation. Full technical description is provided in the supplementary materials. 


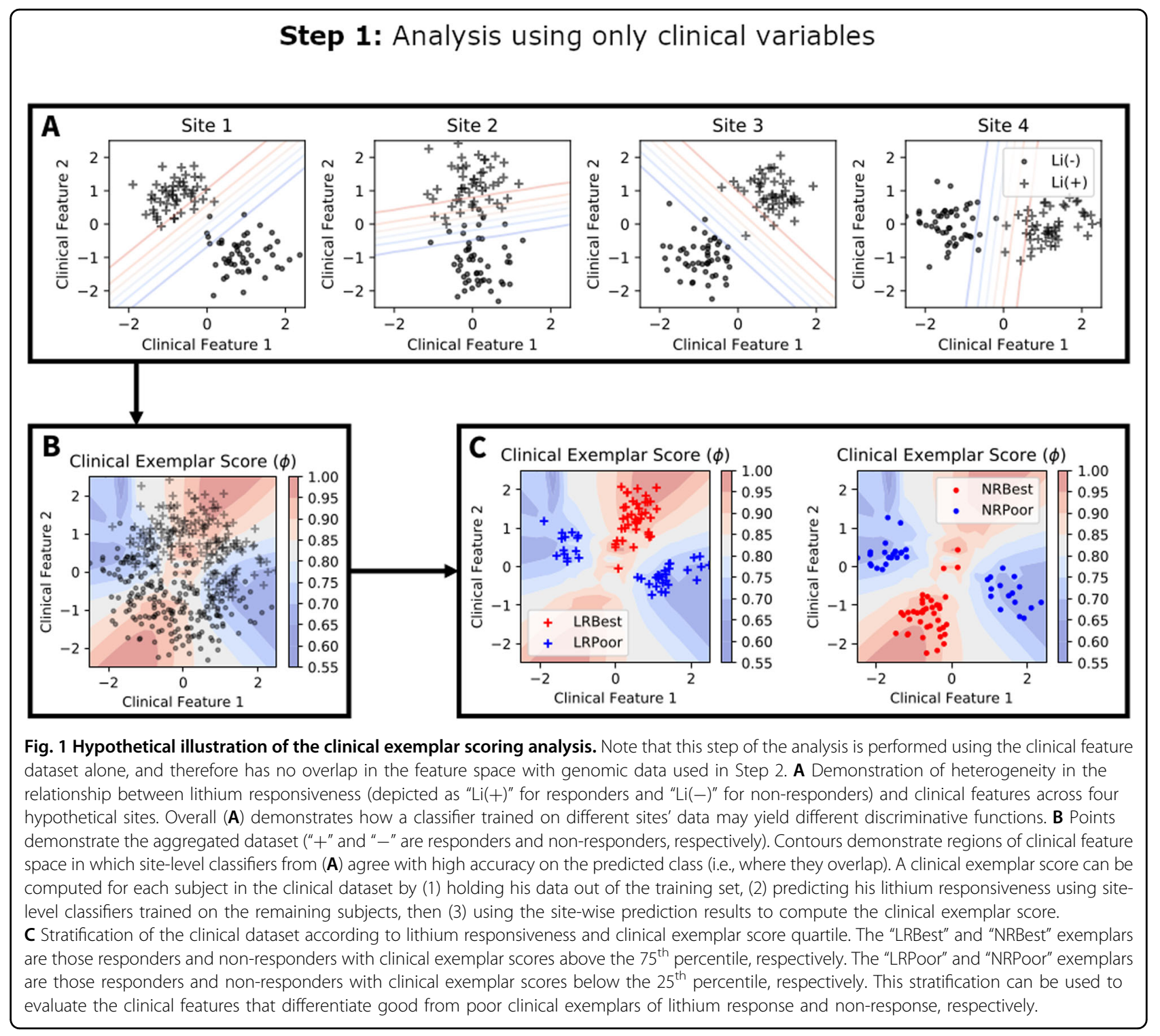

We employed a random forest classifier (RFC) ${ }^{12}$ under the same specifications as in Nunes et al. ${ }^{5}$ (100 estimators; SciKit Learn implementation ${ }^{13}$ ). Similar to that study, missing data were marginalized by sampling from uninformative priors on respective variables' domains, class imbalance was addressed using Synthetic Minority Oversampling Technique (SMOTE) with a Tomek link function. Briefly, SMOTE augments the minority class in a training set with synthetic but similar observations. The Tomek link removes synthetic observations that overlap in feature space with the majority class, or are close to a decision boundary ${ }^{14}$. Furthermore, neither hyperparameter optimization nor further feature set restrictions were undertaken, since hyperparameter optimization does not improve the clinical prediction model $^{5}$ and feature informativeness is taken into account during RFC training.
For each site in the clinical predictors dataset, our predict every subject out (PESO) analysis protocol begins with a leave-one-out cross-validation run to obtain outof-sample predictions for each of that site's constituent subjects. We then train an RFC on that site's data and predict lithium response in all other sites' subjects. Each subject thus obtains one prediction of his or her response for each site's classifier. Accuracy of each site's prediction was calculated as the reciprocal of the absolute error for each subject.

Using the distribution of accuracies recorded for each subject, the clinical exemplar score is computed as an index of both (A) how accurately the subject's response is predicted, and (B) the agreement across sites regarding the subject's class. Poor clinical exemplars will score near zero (poor accuracy and poor agreement across sites). 
Conversely, the best clinical exemplars score near 1 (accurate classification with high agreement across sites). Subjects classified accurately with poor agreement (or vice versa) have intermediate clinical exemplar scores.

\section{Comparing characteristics of the best and poor clinical exemplars in the clinical dataset}

Univariate clinical feature differences were compared between the best clinical exemplars of lithium response and non-response ("LRBest" and "NRBest," respectively; the top $25 \%$ of clinical exemplar scores per class), and the corresponding poor clinical exemplars ("LRPoor" and "NRPoor," respectively; the bottom 25\% of clinical exemplar scores per class). Continuous variables were compared using the two-sample permutation test and categorical variables were compared using the randomization chi-square test (with 10,000 replications). The significance threshold was adjusted for 116 comparisons: $\alpha_{c}=0.05 / 116=0.0004$.

\section{Step 2: Biological validation through genomic classification}

Figure 2 illustrates Step 2 of the present study, wherein we compare the genetic prediction of lithium response when genotyped subjects are stratified by their clinical exemplar scores. Recall that the stratification into "best clinical exemplars" and "poor clinical exemplars" is done exclusively in Step 1 using clinical variables, and therefore absolutely no clinical features are included in the genomic classification analysis. This step of our study uses genomic

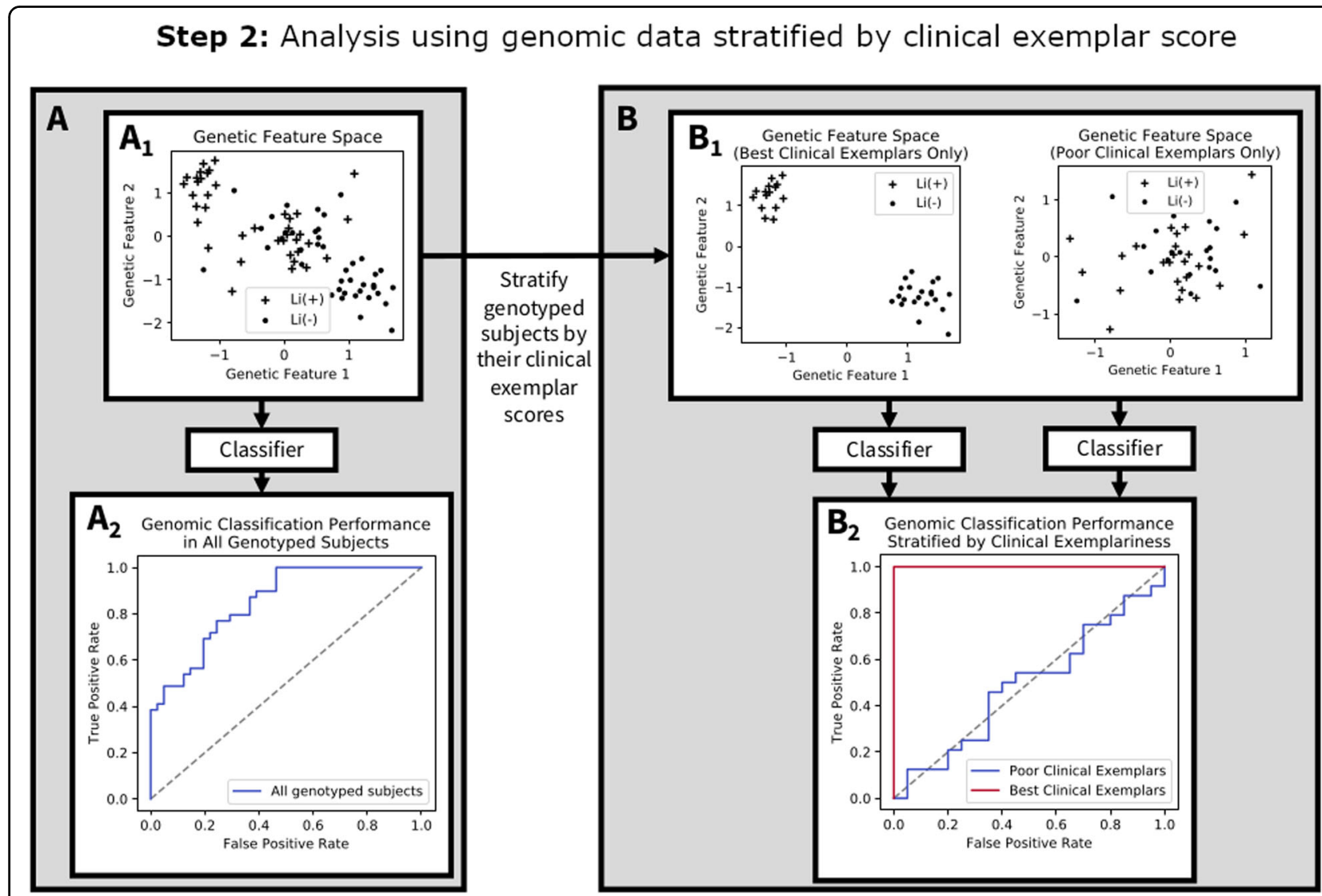

Fig. 2 Hypothetical illustration of Step 2 of this study's analysis, which evaluates the degree to which stratification of the subject group into best and poor clinical exemplars can improve genomic classification performance. A Subjects' genotypes lie on a genotypic feature space (shown in $\left(\mathbf{A}_{\mathbf{1}}\right)$ as a simplified 2 dimensional plane), ( $\mathbf{A}_{\mathbf{2}}$ ) shows a hypothetical ROC curve for these aggregated data. $\mathbf{B}$ Each genotyped subject has a clinical exemplar score computed from Step 1 of the present study. The exemplar score merely identifies the degree to which a subject's clinical profile (i.e., symptoms, family history, comorbidities, etc.) is reliably predictive of lithium responsiveness across sites in the clinical dataset; it merely allows us to define the different groups for the genomic classification analysis (best and poor clinical exemplars); $\mathbf{B}_{\mathbf{1}}$ ) illustrates stratification of the aggregated genotyped sample into the "Best" clinical exemplars (subjects with top 25\% of clinical exemplar scores within each of the responder and non-responder groups, respectively), and the "Poor" clinical exemplars (those with the lowest $25 \%$ of clinical exemplar scores in each responsiveness class). Importantly, the clinical exemplar score is not computed based on any genomic information, nor are any variables from the clinical dataset included in the genomic classification analysis. We then apply classifiers to the genomic data in each of these best and poor clinical exemplar strata, respectively, and compare classification performance $\left(\mathbf{B}_{\mathbf{2}}\right)$. The hypothetical receiver operating characteristic curve in $\left(\mathbf{B}_{\mathbf{2}}\right)$ reflects our hypothesis, that genetic classification of lithium response will be superior among the subgroup of best clinical exemplars. 
data from a relatively ethnically homogeneous subset of subjects in the ConLiGen GWAS ${ }^{10}$ (those sourced from Halifax, Nova Scotia, Canada) who also had detailed clinical information collected for Step 1 of the present study.

\section{Genomic data collection}

Genomic data, obtained as part of the ConLiGen GWAS $^{10}$, were available for 321 of the 1266 subjects whose clinical data were analyzed in Step 1 of our study. In the ConLiGen sample, these subjects were a relatively ethnically homogeneous subset contributed by the Canadian group, which also has a similar number of responders and non-responders (i.e., "class balance", with 159 [49.5\%] responders; Supplementary Table 2). Supplementary Figs. 3 and 4 show that there was no population stratification in our subsample. We restricted the data to only the 47,465 directly genotyped SNPs for which complete data were available across all ConLiGen sites. Preprocessing and quality control were done according to Hou et al. ${ }^{10}$ (see Supplementary Materials).

\section{Genomic classification with stratification by clinical exemplar score}

For genotyped subjects, we compared the performance of a classifier applied to genomic data from (A) all 321 subject's, (B) the poor clinical exemplars', and (C) the best clinical exemplars'. We employed L2-penalized logistic regression $(C=1$ set a priori, since this imposes a consistent prior on model weights, facilitating their comparison). Model criticism was performed under stratified-10-fold cross-validation. In the Supplementary Materials, we provide an alternative protocol using repeated shuffle-split cross-validation, in which we also test the sensitivity of our results to test set size.

Our primary outcome was the average cross-validated Matthews correlation coefficient (MCC). Classification performance differences were compared between conditions using the Kruskal-Wallis test. Where a statistically significant difference was observed (at $\alpha=0.05$ ), pair-wise comparisons were done with the Mann-Whitney U tests (at threshold $\alpha_{c}=0.05 / 3=0.017$ ). We secondarily report accuracy, area under the receiver operating characteristic curve (ROC-AUC), Cohen's kappa, sensitivity, specificity, positive predictive value (PPV), and negative predictive value (NPV).

\section{Gene enrichment analysis}

In the model trained on the best clinical exemplars, we indexed variants whose logistic regression coefficients agreed in sign across all cross-validation folds, then applied the statistical enrichment test to the nearest associated genes using the PANTHER classification system v. $14.1^{15}$. For comparison, we repeated this analysis using logistic regression coefficients from the poor exemplar group. Our significance threshold was $\alpha_{\mathrm{FDR}}=0.05$ where FDR indicates correction for false discovery rate. Further enrichment analysis details are provided in the Supplementary Methods.

\section{Results}

Step 1: Scoring and characterization of clinical exemplars Heterogeneity of classification performance across sites in the predict every subject out analysis

Supplementary Fig. 5 shows that the distributions of accuracy between site-level models were variable in shape and modality, highlighting the heterogeneity in betweensite classification behavior whose redress is the central motivating factor of our present study.

\section{Characteristics of the best and poor clinical exemplars in the clinical dataset}

Within the clinical dataset of Step 1, there were 110 individuals in LRBest and LRPoor groups, and 207 individuals in the NRBest and NRPoor groups (Table 1). Note that the groups compared in Table 1 are not those for whom we attempted genomic classification in Step 2 of the present study (those comparisons are presented in Supplementary Table 2). The LRBest group came predominantly from IGSLi (53.6\%) and Ontario (21.8\%), and most NRBest subjects were from Maritimes $(72.5 \%)$ and Montreal (25.1\%).

The LRBest group showed a later age of onset (median $28 \mathrm{y}$, interquartile range, $\operatorname{IQR}[21,36])$ compared to NRBest (median 19, IQR; $[16,24] p<0.00001$ ).

All the LRBest subjects for whom clinical course information was available showed a completely episodic course, whereas NRBest courses were mainly chronic fluctuating (43.5\%) and episodic with residual symptoms (44.9\%; omnibus $p=0.0001$ ). Interestingly, NRPoor subjects had predominantly completely episodic clinical courses (74.1\%), whereas LRPoor subjects exhibited predominantly chronic fluctuating (41\%) and chronic (25.3\%) courses, with only $18.1 \%$ being completely episodic (omnibus $p=0.0001$ ).

The complete absence of rapid cycling was reported in 98.3\% of LRBest, and $47.2 \%$ of NRBest $(p=0.0001)$. The majority of NRBest subjects (49.2\%) reported spontaneous rapid cycling.

History of lifetime psychosis differed between LRBest and NRBest, with a total of $42.8 \%$ of the non-responders reporting episodic and mood congruent psychosis (compared to only $16.7 \%$ of responders; $p=0.0001$ ). Nonresponders also reported incongruent episodic psychosis in $18.6 \%$ of cases, with only $37.1 \%$ of non-responders reporting an absence of psychosis altogether. In contrast, $83.3 \%$ of the best clinical exemplars of lithium response 
Table 1 Characteristics of the best (upper $25 \%$ of exemplar scores) and poor (lower $25 \%$ of exemplar scores) exemplars of lithium response and non-response, respectively.

\begin{tabular}{|c|c|c|c|c|c|c|}
\hline & \multicolumn{3}{|l|}{ Best exemplars } & \multicolumn{3}{|l|}{ Poor exemplars } \\
\hline & Non-responder & Responder & $p$ & Non-responder & Responder & $p$ \\
\hline$n$ & 207 & 110 & & 207 & 110 & \\
\hline Male (\%) & $76(36.7)$ & $46(41.8)$ & 0.39826 & 79 (38.2) & 34 (30.9) & 0.21488 \\
\hline Age (y) & $42.44[32.12,51.80]$ & $54.19[42.38,65.46]$ & $<0.00001$ & $45.92[36.39,57.80]$ & $59.74[44.35,66.12]$ & $<0.00001$ \\
\hline Center (\%) & & & - & & & 0.0001 \\
\hline CU & $4(1.9)$ & $21(19.1)$ & & $74(35.7)$ & $1(0.9)$ & \\
\hline CB & $1(0.5)$ & $0(0.0)$ & & 70 (33.8) & $11(10.0)$ & \\
\hline IGSLi & $0((0.0)$ & $59(53.6)$ & & $0(0.0)$ & $8(7.3)$ & \\
\hline MAR & $150(72.5)$ & $6(5.5)$ & & $38(18.4)$ & $16(14.5)$ & \\
\hline MTL & $52(25.1)$ & $0(0.0)$ & & $11(5.3)$ & $2(1.8)$ & \\
\hline ON & $0(0.0)$ & $24(21.8)$ & & $6(2.9)$ & $21(19.1)$ & \\
\hline POZ & $0(0.0)$ & $0(0.0)$ & & $8(3.9)$ & $51(46.4)$ & \\
\hline Diagnosis (\%) & & & 0.12369 & & & 0.0472 \\
\hline BD I & $139(67.1)$ & $71(64.5)$ & & $136(65.7)$ & $66(60.0)$ & \\
\hline BD ॥ & $62(30.0)$ & $33(30.0)$ & & $51(24.6)$ & $36(32.7)$ & \\
\hline MDD recurrent & $0(0.0)$ & $3(2.7)$ & & $3(1.4)$ & $4(3.6)$ & \\
\hline MDD single & & & & $0(0.0)$ & $1(0.9)$ & \\
\hline SZA & $6(2.9)$ & $3(2.7)$ & & $17(8.2)$ & $3(2.7)$ & \\
\hline Age of onset (y) & $19.00[16.00,24.00]$ & $28.00[21.00,36.00]$ & $<0.00001$ & $22.50[18.00,32.25]$ & $27.50[18.25,35.00]$ & 0.16618 \\
\hline Onset of depression (y) & $20.00[16.00,25.00]$ & $30.00[23.00,37.00]$ & $<0.00001$ & $28.00[20.00,38.00]$ & $30.00[20.50,37.50]$ & 0.77534 \\
\hline Onset of mania (y) & $25.00[21.00,32.00]$ & $30.00[26.00,40.00]$ & 0.00012 & $29.33[22.00,36.50]$ & $32.00[28.00,39.75]$ & 0.00928 \\
\hline Onset of hypomania (y) & $26.50[21.00,38.50]$ & $38.00[25.50,45.50]$ & 0.00293 & $32.49(14.59)$ & $38.13(12.16)$ & 0.06037 \\
\hline Polarity episode 1 (\%) & & & 0.0002 & & & 0.011 \\
\hline Biphasic (D-M) & $4(2.0)$ & $5(5.8)$ & & $3(5.8)$ & $1(2.4)$ & \\
\hline Biphasic (M-D) & $13(6.6)$ & $4(4.7)$ & & $2(3.8)$ & $2(4.8)$ & \\
\hline Hypomania & $19(9.7)$ & $8(9.3)$ & & $10(19.2)$ & $3(7.1)$ & \\
\hline Major depression & $142(72.4)$ & $42(48.8)$ & & $20(38.5)$ & $30(71.4)$ & \\
\hline Mania & $13(6.6)$ & $16(18.6)$ & & $16(30.8)$ & $4(9.5)$ & \\
\hline Minor depression & $5(2.6)$ & $11(12.8)$ & & $1((1.9)$ & $2(4.8)$ & \\
\hline Clinical course (\%) & & & 0.0001 & & & 0.0001 \\
\hline Chronic & $14(6.8)$ & $0(0.0)$ & & $8(4.1)$ & $21(25.3)$ & \\
\hline Chronic deteriorating & $2(1.0)$ & $0(0.0)$ & & $3(1.5)$ & $2(2.4)$ & \\
\hline Chronic fluctuating & $90(43.5)$ & $0(0.0)$ & & $11(5.6)$ & $34(41.0)$ & \\
\hline Completely episodic & $7(3.4)$ & $27(100.0)$ & & $146(74.1)$ & $15(18.1)$ & \\
\hline Continuous cycling & $1(0.5)$ & $0(0.0)$ & & $7(3.6)$ & $2(2.4)$ & \\
\hline Episodic with residual & $93(44.9)$ & $0(0.0)$ & & $22(11.2)$ & $9(10.8)$ & \\
\hline Lifetime manias, $n$ & $3.00[1.00,7.00]$ & $2.00[0.00,3.00]$ & 0.00001 & $3.00[1.00,6.00]$ & $2.00[1.00,3.00]$ & 0.02059 \\
\hline Lifetime depressions, $n$ & $5.00[3.00,15.00]$ & $3.00[2.00,6.00]$ & $<0.00001$ & $4.00[2.00,8.00]$ & $4.00[2.00,6.00]$ & 0.03006 \\
\hline
\end{tabular}


Table 1 continued

\begin{tabular}{|c|c|c|c|c|c|c|}
\hline & \multicolumn{3}{|l|}{ Best exemplars } & \multicolumn{3}{|l|}{ Poor exemplars } \\
\hline & Non-responder & Responder & $p$ & Non-responder & Responder & $p$ \\
\hline Lifetime mixed, $n$ & $0.00[0.00,1.00]$ & $0.00[0.00,0.00]$ & $<0.00001$ & $0.00[0.00,0.00]$ & $0.00[0.00,0.00]$ & 0.40342 \\
\hline Lifetime multiphasic, $n$ & $0.00[0.00,1.00]$ & $0.00[0.00,2.00]$ & 0.00006 & $0.00[0.00,0.00]$ & $0.00[0.00,0.00]$ & 0.18415 \\
\hline Total lifetime episodes, $n$ & $9.00[5.00,24.50]$ & $6.00[5.00,10.00]$ & $<0.00001$ & $8.00[5.00,15.00]$ & $5.00[4.00,9.00]$ & 0.00464 \\
\hline Rapid cycling (\%) & & & 0.0001 & & & 0.70113 \\
\hline Never & $92(47.2)$ & $59(98.3)$ & & $56(93.3)$ & $80(96.4)$ & \\
\hline Only on antidepressants & $7(3.6)$ & $0(0.0)$ & & $2(3.3)$ & $1(1.2)$ & \\
\hline Spontaneous & $96(49.2)$ & $1(1.7)$ & & $2(3.3)$ & $2(2.4)$ & \\
\hline Rapid mood switching (\%) & $47(63.5)$ & $0(0.0)$ & 0.06059 & $6(21.4)$ & $1(1.8)$ & 0.0049 \\
\hline Lifetime psychosis (\%) & & & 0.0001 & & & 0.0018 \\
\hline In episodes, congruent & $83(42.8)$ & $5(16.7)$ & & $51(38.9)$ & $15(20.0)$ & \\
\hline In episodes, incongruent & $36(18.6)$ & $0(0.0)$ & & $8(6.1)$ & $1(1.3)$ & \\
\hline Never & $72(37.1)$ & $25(83.3)$ & & $70(53.4)$ & $59(78.7)$ & \\
\hline Outside of mood episodes & $3(1.5)$ & $0(0.0)$ & & $2(1.5)$ & $0(0.0)$ & \\
\hline GAF at last assessment & $70.00[55.00,75.00]$ & $90.00[90.00,95.00]$ & $<0.00001$ & $75.00[60.00,86.25]$ & $87.50[80.00,90.00]$ & 0.01251 \\
\hline Li total score & $2.00[0.00,4.00]$ & $8.00[8.00,10.00]$ & $<0.00001$ & $3.00[1.00,5.00]$ & $8.00[7.00,9.00]$ & $<0.00001$ \\
\hline Episodes on Li, $n$ & $4.00[1.25,10.00]$ & $0.00[0.00,1.75]$ & 0.01189 & $2.00[1.00,4.00]$ & $1.00[0.00,1.50]$ & 0.00008 \\
\hline Episodes pre-Li, $n$ & $4.00[3.00,12.00]$ & $5.00[4.00,15.75]$ & 0.14411 & $4.00[3.00,7.00]$ & $4.00[3.00,6.00]$ & 0.77478 \\
\hline SA, $n$ & $0.00[0.00,1.00]$ & $0.00[0.00,0.00]$ & 0.00254 & $0.00[0.00,0.00]$ & $0.00[0.00,0.00]$ & 0.15478 \\
\hline Potentially lethal SA, $n$ & $1.00[0.00,1.00]$ & $0.00[0.00,0.00]$ & 0.00033 & $0.00[0.00,0.00]$ & $0.00[0.00,1.00]$ & 0.00502 \\
\hline Age at SA1 (\%) & $26.00[17.00,35.00]$ & $20.00[18.00,36.00]$ & 0.75222 & $36.16(13.87)$ & 33.79 (12.08) & 0.67026 \\
\hline FDR mood disorder (\%) & $99(55.3)$ & $31(35.2)$ & 0.0027 & $76(73.8)$ & $22(40.0)$ & 0.0002 \\
\hline FDR bipolar disorder (\%) & $44(21.7)$ & $9(10.1)$ & 0.0214 & $62(51.2)$ & $42(39.3)$ & 0.08039 \\
\hline FDR BD-I, $n$ & $0.00[0.00,0.00]$ & $0.00[0.00,0.00]$ & 0.00338 & $0.00[0.00,1.00]$ & $0.00[0.00,1.00]$ & 0.05523 \\
\hline FDR BD-II, $n$ & $0.00[0.00,0.00]$ & $0.00[0.00,0.00]$ & 0.7157 & $0.00[0.00,0.00]$ & $0.00[0.00,0.00]$ & 0.89852 \\
\hline FDR unipolar $D, n$ & $1.00[0.00,1.00]$ & $0.00[0.00,1.00]$ & 0.00515 & $0.00[0.00,1.00]$ & $0.00[0.00,1.00]$ & 0.5521 \\
\hline FDR SZA, $n$ & $0.00[0.00,0.00]$ & $0.00[0.00,0.00]$ & 0.72095 & $0.00[0.00,0.00]$ & $0.00[0.00,0.00]$ & 0.7671 \\
\hline FDR SCZ, $n$ & $0.00[0.00,0.00]$ & $0.00[0.00,0.00]$ & 0.05084 & $0.00[0.00,0.00]$ & $0.00[0.00,0.00]$ & 0.21199 \\
\hline FDR anxiety, $n$ & $0.00[0.00,0.00]$ & $0.00[0.00,0.00]$ & 0.00069 & $0.00[0.00,0.00]$ & $0.00[0.00,0.00]$ & 0.32271 \\
\hline FDR unaffected, $n$ & $0.00[0.00,1.00]$ & $0.00[0.00,0.00]$ & 0.00042 & $3.50[0.00,7.00]$ & $0.00[0.00,0.00]$ & $<0.00001$ \\
\hline FDR suicide, $n$ & $0.00[0.00,0.00]$ & $0.00[0.00,0.00]$ & 0.68103 & $0.00[0.00,0.00]$ & $0.00[0.00,0.00]$ & 0.86527 \\
\hline FDR SA, $n$ & $0.00[0.00,0.00]$ & $0.00[0.00,0.00]$ & 0.22235 & $0.00[0.00,0.00]$ & $0.00[0.00,0.00]$ & 0.07323 \\
\hline SDR suicide, $n$ & $0.00[0.00,0.00]$ & $0.00[0.00,0.00]$ & 0.36643 & $0.00[0.00,0.00]$ & $0.00[0.00,0.00]$ & 0.66814 \\
\hline SDR SA, $n$ & $0.00[0.00,0.00]$ & $0.00[0.00,0.00]$ & 0.26629 & $0.00[0.00,0.00]$ & $0.00[0.00,0.00]$ & 0.68636 \\
\hline Mood polarity at suicide attempt (\%) & & & 0.33847 & & & 1 \\
\hline Major depression & $74(91.4)$ & $3(75.0)$ & & $0(0.0)$ & $0(0.0)$ & \\
\hline Mania & $3(3.7)$ & $1(25.0)$ & & $3(16.7)$ & $0(0.0)$ & \\
\hline Minor depression & $1(1.2)$ & $0(0.0)$ & & $0(0.0)$ & $0(0.0)$ & \\
\hline
\end{tabular}


Table 1 continued

\begin{tabular}{|c|c|c|c|c|c|c|}
\hline & \multicolumn{3}{|l|}{ Best exemplars } & \multicolumn{3}{|l|}{ Poor exemplars } \\
\hline & Non-responder & Responder & $p$ & Non-responder & Responder & $p$ \\
\hline Mixed & $2(2.5)$ & $0(0.0)$ & & $0(0.0)$ & $0(0.0)$ & \\
\hline Rapid cycling & $1(1.2)$ & $0(0.0)$ & & $0(0.0)$ & $0(0.0)$ & \\
\hline LT Hx SI (\%) & $114(61.3)$ & $18(34.0)$ & 0.0007 & $61(44.2)$ & $11(40.7)$ & 0.82612 \\
\hline SI related to mood episode (\%) & & & 1 & & & - \\
\hline No & $1(0.9)$ & $0(0.0)$ & & $0(0.0)$ & $0(0.0)$ & \\
\hline Sometimes, not always & $6(5.7)$ & $0(0.0)$ & & $0(0.0)$ & $0(0.0)$ & \\
\hline Yes & $99(93.4)$ & $2(100.0)$ & & $9(100.0)$ & $8(100.0)$ & \\
\hline Social anxiety disorder (\%) & $54(26.6)$ & $0(0.0)$ & 0.0007 & $8(4.5)$ & $26(35.6)$ & 0.0001 \\
\hline Panic disorder (\%) & $57(27.9)$ & $2(2.1)$ & 0.0001 & $28(15.5)$ & $43(48.9)$ & 0.0001 \\
\hline GAD (\%) & $84(41.2)$ & $1(3.6)$ & 0.0001 & $13(7.3)$ & $39(52.0)$ & 0.0001 \\
\hline OCD (\%) & $29(14.1)$ & $2(2.1)$ & 0.0025 & $1(0.6)$ & $8(9.2)$ & 0.0004 \\
\hline Substance abuse (\%) & $78(37.9)$ & $2(2.0)$ & 0.0001 & $43(21.0)$ & $39(41.5)$ & 0.0005 \\
\hline ADHD (\%) & $11(5.5)$ & $0(0.0)$ & 1 & $11(10.6)$ & $45(60.8)$ & 0.0001 \\
\hline Learning disability (\%) & $9(4.5)$ & $0(0.0)$ & 1 & $11(10.6)$ & $38(51.4)$ & 0.0001 \\
\hline Primary insomnia (\%) & $35(17.5)$ & $0(0.0)$ & 0.38046 & $7(6.7)$ & $9(11.8)$ & 0.28727 \\
\hline Personality disorder (\%) & $38(19.1)$ & $0(0.0)$ & 0.37536 & $3(3.4)$ & $23(31.9)$ & 0.0001 \\
\hline Diabetes mellitus (\%) & $20(10.3)$ & $0(0.0)$ & 0.59934 & $6(8.3)$ & $5(7.5)$ & 1 \\
\hline HTN (\%) & $22(11.4)$ & $2(20.0)$ & 0.61024 & $17(23.6)$ & $35(53.0)$ & 0.0006 \\
\hline Menstrual abnormality (\%) & $39(34.2)$ & $3(60.0)$ & 0.34787 & $8(26.7)$ & $2(4.7)$ & 0.0144 \\
\hline Thyroid disease (\%) & $55(29.3)$ & $2(33.3)$ & 1 & $18(32.1)$ & $8(11.9)$ & 0.0082 \\
\hline Head injury (\%) & $48(27.0)$ & $1(20.0)$ & 1 & $17(34.0)$ & $24(39.3)$ & 0.69833 \\
\hline Migraine (\%) & $44(23.5)$ & $2(33.3)$ & 0.62184 & $11(19.3)$ & $9(13.8)$ & 0.47385 \\
\hline SES (\%) & & & 0.0001 & & & 0.0001 \\
\hline Disabled & $65(36.3)$ & $1(3.4)$ & & $6(3.4)$ & $3(4.0)$ & \\
\hline Other & $12(6.7)$ & $8(27.6)$ & & $23(13.2)$ & $0(0.0)$ & \\
\hline Retired & $8(4.5)$ & $7(24.1)$ & & $25(14.4)$ & $22(29.3)$ & \\
\hline Social assistance & $32(17.9)$ & $2(6.9)$ & & $4(2.3)$ & $3(4.0)$ & \\
\hline Unemployment insurance & $18(10.1)$ & $0(0.0)$ & & $7(4.0)$ & $3(4.0)$ & \\
\hline Unknown & $2(1.1)$ & $1(3.4)$ & & $1(0.6)$ & $0(0.0)$ & \\
\hline Work full-time & $30(16.8)$ & $10(34.5)$ & & $96(55.2)$ & $29(38.7)$ & \\
\hline Work part-time & $12(6.7)$ & $0(0.0)$ & & $12(6.9)$ & $15(20.0)$ & \\
\hline Marital status (\%) & & & 0.0001 & & & 0.0492 \\
\hline Divorced & $47(23.3)$ & $2(6.7)$ & & $16(8.1)$ & $9(11.0)$ & \\
\hline Married & $84(41.6)$ & $19(63.3)$ & & $118(59.6)$ & $51(62.2)$ & \\
\hline Single & 67 (33.2) & $2(6.7)$ & & $51(25.8)$ & $11(13.4)$ & \\
\hline Widowed & $4(2.0)$ & $7(23.3)$ & & $13(6.6)$ & $11(13.4)$ & \\
\hline
\end{tabular}

Categorical data are presented as count (\%), whereas normally distributed continuous variables are presented as mean (standard deviation), and non-normal continuous variables are presented as median [interquartile range]. CU Cagliari (University), CB Cagliari (Centro Bini), IGSLi International Group for the Study of Lithium, MAR Maritimes, MTL Montreal, ON Ontario, POZ Poznan, BD bipolar disorder, MDD major depressive disorder, SZA schizoaffective disorder, GFA global assessment of functioning, $L i$ lithium, $S A$ suicide attempts, FDR first degree relatives, SDR second degree relatives, SCZ schizophrenia, $S /$ suicidal ideation, $H x$ history, GAD generalized anxiety disorder, $O C D$ obsessive compulsive disorder, ADHD attention deficit hyperactivity disorder, HTN hypertension, SES socioeconomic status. 


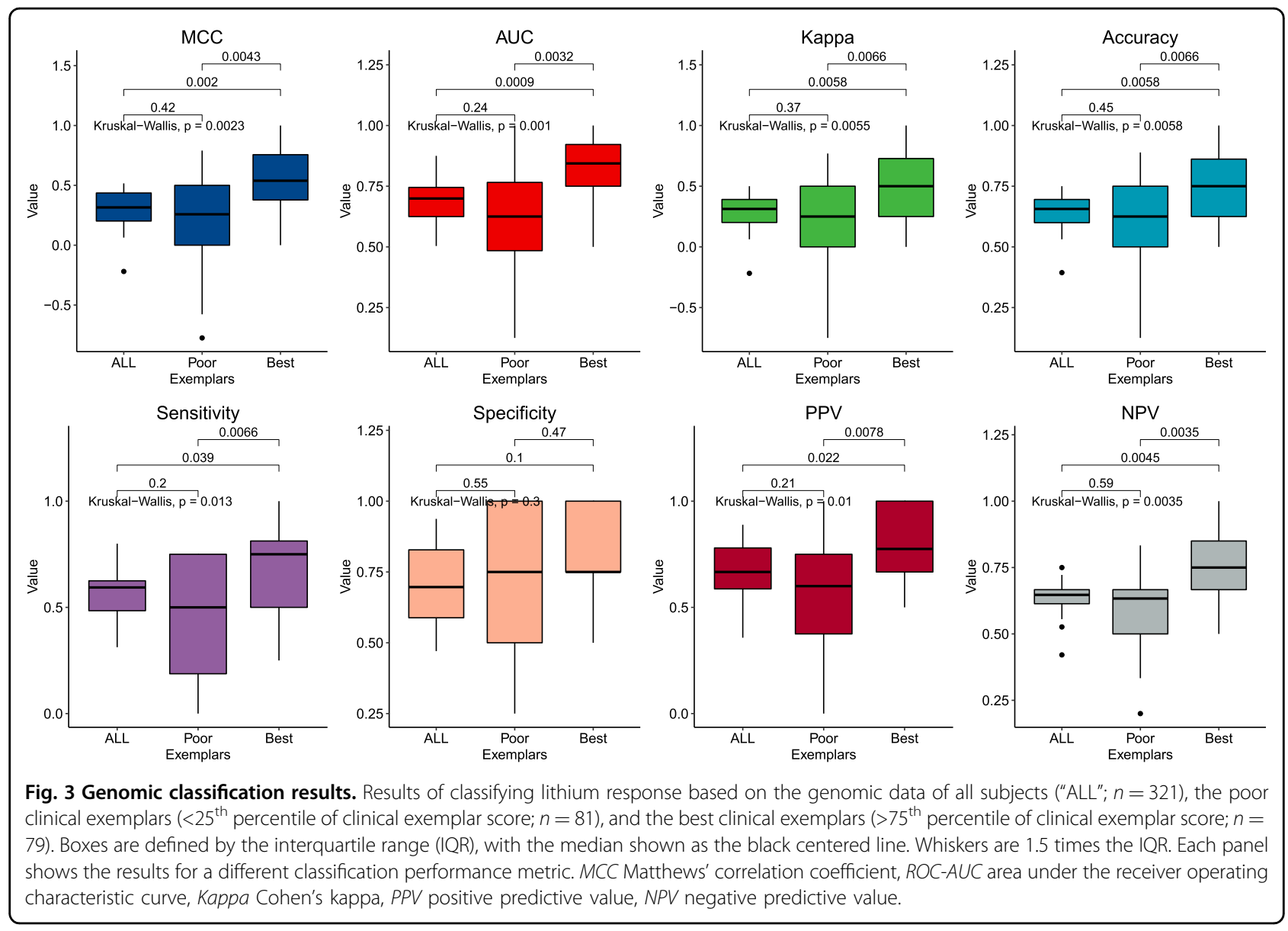

reported a complete absence of lifetime psychosis. There was also a general trend toward lower rates of psychiatric comorbidity in LRBest compared to the NRBest group, whereas LRPoor generally showed more psychiatric comorbidity than NRPoor subjects.

\section{Step 2: Biological validation through genomic classification}

Recall that the genomic data for this element of the analysis are derived from a single site in the ConLiGen data (Dalhousie University, Canada). Supplementary Figs. 3 and 4 demonstrate relative lack of genomic population stratification in this subset, with a comparison to the broader ConLiGen sample.

\section{Genomic classification with stratification by clinical exemplar score}

Genotyped subjects overlapped with clinical data from the Maritimes $(n=129 ; 40 \%)$, Montreal $(n=74 ; 23 \%)$, Ontario $(n=62 ; 19 \%)$, and IGSLi $(n=56 ; 17 \%)$, although in the ConLiGen GWAS ${ }^{8}$, they were all classified as from the Maritimes (Dalhousie University, Canada). It bears repeating that none of the 321 genotyped subjects in the present study came from the relatively genetically distinct Sardinian population whose clinical characteristics were evaluated in Step 1 of our study. Notwithstanding, most clinical differences reflect those reported in Table 1 and thus are reported in Supplementary Table 2.

Genomic classification results are presented in Fig. 3 and in Supplementary Table 3. The median MCC for classification of the best clinical exemplars was 0.58 (IQR $[0.41,0.77])$, which was greater than classification analyses with either the poor clinical exemplars $(0.29[0.06,0.5]$; $p=0.0043)$, or the entire dataset $(0.32[0.2,0.44] ; p=$ 0.002). The ROC-AUC for classification of lithium response in the best clinical exemplars was $0.88[0.83$, $0.98]$, which was greater than that of the model trained only on poor clinical exemplars $(0.66[0.61,0.80] ; p=$ $0.0032)$ or the whole dataset $(0.7[0.62,0.75] ; p=0.001)$.

\section{Gene enrichment analysis}

Figure 4 shows pathway analysis results for the best exemplar stratum of the genotyped subjects. Enriched pathways involved (A) muscarinic acetylcholine receptor types 1 and 3 signaling (mAChR1/3; 27 genes, false discovery rate $\mathrm{FDR}=0.017$ ), (B) Alzheimer disease-amyloid 


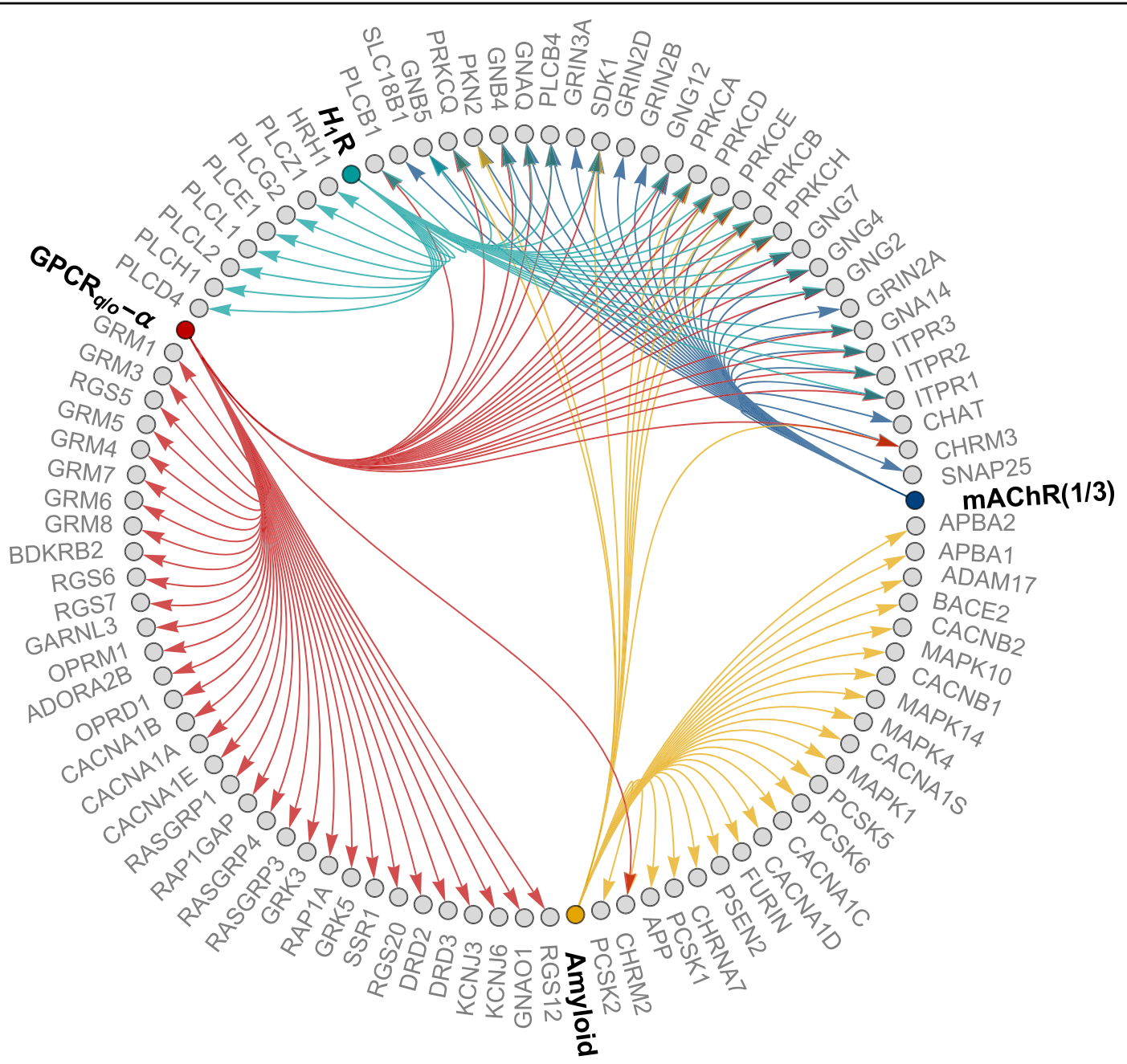

\begin{tabular}{|l|l|l|l|l|}
\hline Pathway & $\boldsymbol{N}_{\text {genes }}$ & $\mathbf{I}$ & $\mathbf{p}$ & FDR \\
\hline$\square$ Muscarinic $\mathrm{AChR}_{1,3}$ signaling pathway & 27 & + & 0.0001 & 0.017 \\
\hline$\square$ Alzheimer disease-amyloid secretase pathway & 30 & + & 0.0005 & 0.034 \\
\hline$\square$ Heterotrimeric $\mathrm{GPCR}_{q / \mathrm{o}} \alpha$ signaling pathway & 53 & + & 0.0008 & 0.041 \\
\hline$\square$ Histamine $H_{1} R$ signaling pathway & 27 & + & 0.001 & 0.039 \\
\hline
\end{tabular}

Fig. 4 Gene enrichment in the best clinical exemplars. Results of the statistical enrichment test using the logistic regression coefficients from the classifier trained on the best exemplar stratum. Individual genes are shown in gray, with pathway nodes (and edges) colored according to the pathway identity. The terms in bold along the perimeter of the graph (i.e., "Amyloid," " $H_{1} R$ ", "GPCR $/ \mathrm{o}^{-} a$ ", and "mAChR(1/3)") are pathway names, with edges connected to their constituent genes. AChR acetylcholine receptor, GPCR G-protein-coupled receptor, HIR histamine H1 receptor, FDR false discovery rate.

secretase (30 genes, $\mathrm{FDR}=0.034)$, $(\mathrm{C})$ heterotrimeric $\mathrm{G}$ protein-coupled receptor Gq/Go $\alpha$ signaling (GPCRq/o- $\alpha$; 53 genes, $\mathrm{FDR}=0.04)$, and $(\mathrm{D})$ histamine H1R mediated signaling (H1R; 27 genes, FDR =0.039). Complete gene set analysis results are shown in Supplementary Table 4. Enrichment studies in the gene ontology "cellular component" and "biological function" categories are shown in Supplementary Tables 5 and 6.

\section{Discussion}

This paper has demonstrated that the best clinical exemplars of lithium response and non-response may be 
more genetically distinct than their less exemplary counterparts, particularly in genes related to GPCRq/o- $\alpha$, $\mathrm{mAChR} 1 / 3$, or H1R signaling, and the Alzheimer's amyloid-secretase pathway. Clinical exemplars' clinical profiles were distinct and consistent with past phenotypic research on lithium responders. The genetic separability of clinical exemplars of lithium response and nonresponse confers some biological validity upon the practice of detailed clinical evaluation, whose predictive utility we have previously demonstrated ${ }^{5}$.

One of our most important findings was characterization of the LRBest group as individuals with generally (A) completely episodic clinical course, (B) few psychiatric comorbidities, (C) later age of onset, (D) absence of rapid cycling, and (E) either absence of psychosis or limitation to mood congruent intra-episodic form. The first two findings are likely the strongest since we observe the opposite pattern among the LRPoor and NRPoor groups. Notwithstanding, all of these elements support past evidence on the clinical phenotype of lithium responsive $\mathrm{BD}^{6,16,17}$. For instance, Passmore et al. ${ }^{18}$ found that lithium responders generally had a more episodic course of illness, whereas lamotrigine responders were more likely to have experienced rapid cycling, a higher rate of psychiatric comorbidity, and an earlier age of onset. A later age of onset in lithium responders has been demonstrated in meta-analysis ${ }^{19,20}$. Absence of rapid cycling and psychosis have also been associated with good lithium response ${ }^{20-23}$.

The present study provides strong data-driven support for the idea that a biomarker's utility is contingent upon the application to patients whose clinical presentations are consistent with the condition being targeted ${ }^{24}$. Furthermore, our study provides biological support for the predictive validity of "classical" clinical pictures of lithium response and non-response ${ }^{5,6,16,17}$. The best clinical exemplars of lithium response and non-response were genetically discriminated with a ROC-AUC of 0.88 (95\% CI $[0.83,0.98])$, whereas we found only a ROC-AUC of 0.66 among poor clinical exemplars (95\% CI $[0.61,0.80] ; p$ $=0.0032$ ). Although the absolute classification performance estimates and their generalizability must be tested on larger, more genetically heterogeneous samples that include distinct subpopulations (for example, Sardinians), our main finding remains important: if there was no biologically mediated information in the exemplary phenotype of lithium response (and non-response), then this difference would not have been observed.

Variants most informative in discrimination of the best exemplars showed enrichment of genes involved in the heterotrimeric GPCRq/o- $\alpha$, mAChR1/3, or H1R signaling, and the Alzheimer's amyloid-secretase pathway. Lithium response and BD have long been associated with GPCR signaling $^{25}$. In particular, lithium may affect signaling in both the Go-alpha pathway (at least via adenylate cyclase) and the Gq-alpha pathway (via effects on 1,4,5-triphosphate and protein kinase $\mathrm{C}, \mathrm{PKC})^{26-29}$. Interestingly, our results imply that differences in GPCR signaling may be segregated according to medication responsiveness. Enrichment in the Alzheimer's amyloid-secretase pathway is interesting given the growing interest in the effects of lithium on Alzheimer's pathology. While no significant signal was attributed to the frequently studied gene for GSK3B in the pathway analysis, it was involved in the statistically significant enrichment of the glutamatergic synapse cellular component (Supplementary Table 5). It should be noted that the stabilizing effect of lithium likely involves multiple mechanisms, including chronobiological regulations or microRNAs ${ }^{30-34}$. Some of these findings reflect changes in gene expression rather than DNA sequence variation and thus are not directly comparable with our results. In future work, it would be of interest to characterize a more fine-grained "gradient" of genetic differences across the spectrum of clinical exemplar scores.

An important limitation of our study includes the relatively low sample size for the genomic analysis, whose redress is of particular urgency owing to the relatively strong classification performance (AUC 0.88 [0.83, 0.98]). Indeed, small sample sizes in multi-site ML studies may be associated with inflated classification performance ${ }^{35}$, although large counterexamples exist $\mathrm{t}^{5,36}$. However, our main finding was likely robust to the sample size limitation itself (Supplementary Figs. 6-8). Notwithstanding, we must endeavor to collect detailed clinical information and genotype more patients in our genomic and clinical databases, respectively. As features, our study also used only those SNPs that overlapped across genotyping platforms in the ConLiGen dataset. Unfortunately, however, the number of fully imputed variants was in the order of millions, which would be analytically intractable in the present context. Further discussion of this point is provided in the Supplementary Materials, suffice to say that further methodological work must develop ML methods capable of handling genotypes of 1-10 million SNPs in size.

Our study is also limited by its focus on lithium response, at the exclusion of other mood stabilizers. It is therefore possible, our lithium responders are simply those with a more generally responsive or "less severe" form of BD. The only way to prove specificity would be to obtain data showing a single subject's non-response to other mood stabilizers and response to lithium, but there is evidence that excellent response to lithium may be exclusive to that medication ${ }^{37}$. Despite their propensity for completely episodic clinical course, lithium responders may also have very severe acute episodes. Finally, individuals with few episodes or infrequent episodes would have lower Alda scores by virtue of the "B"-subscale ${ }^{38}$. It will be of great interest to examine exemplar-based genomic classification of mood stabilizer response more 
broadly. We are presently collecting clinical and genomic data for patients treated with other mood stabilizers, but these are not yet as abundant as our lithium response data. However, based on our analyses presented here and in our earlier paper ${ }^{5}$, we would (A) recommend lithium as the first-line mood stabilizer for patients with the profile corresponding to exemplary responders ("classical," Kraepelinean-type BD), (B) not recommend lithium for patients with the characteristics of exemplary nonresponders, and $(\mathrm{C})$ consider a time-limited trial of lithium for patients with information insufficient to classify them into either of these two groups.

\section{Acknowledgements}

Genome Canada/Genome Atlantic (M.A., A.N., R.U.), Dalhousie Department of Psychiatry Research Fund (M.A., A.N.), Dalhousie Medical Research Foundation and the Lindsay Family (M.A., A.N.), Canadian Institutes of Health Research \#166098 (M.A., A.N., R.U.), Canada Research Chairs Program \#231397 (R.U.), Nova Scotia Health Research Foundation Scotia Scholars Graduate Scholarship (A.N.), Killam Postgraduate Scholarship (A.N.), EMBED-BMBF-01EW1904 (MDCR).

\begin{abstract}
Author details
${ }^{1}$ Department of Psychiatry, Dalhousie University, Halifax, NS, Canada. ${ }^{2}$ Faculty of Computer Science, Dalhousie University, Halifax, NS, Canada. ${ }^{3}$ Unit of Clinical Pharmacology \& San Giovanni di Dio Hospital, University Hospital of Cagliari, Cagliari, Italy. ${ }^{4}$ Charité University Medical Center, Campus Charité Mitte, Institute for Social Medicine, Epidemiology and Health Economics, Berlin, Germany. ${ }^{5}$ Department of Biomedical Sciences, Section of Neuroscience and Clinical Pharmacology, University of Cagliari, Cagliari, Italy. ${ }^{6}$ Institute of Human Genetics, University of Bonn, School of Medicine \& University Hospital Bonn, Bonn, Germany. ${ }^{7}$ Centre for Human Genetics, University of Marburg, Marburg, Germany. ${ }^{8}$ Department of Biomedicine, University of Basel, Basel, Switzerland. ${ }^{9}$ Mood Disorders Center of Ottawa, Ottawa, Ontario, Canada. ${ }^{10}$ Department of Psychiatry, University of Toronto, Toronto, Ontario, Canada. ${ }^{11}$ Department of Medical Sciences and Public Health, Section of Psychiatry, University of Cagliari, Cagliari, Italy. ${ }^{12}$ Department of Pharmacology, Dalhousie University, Halifax, Nova Scotia, Canada. ${ }^{13}$ Department of Psychiatry, University of Wurzburg, Wurzburg, Germany. ${ }^{14}$ National Institute of Mental Health, Bethesda, MD, USA. ${ }^{15}$ Charité Universitätsmedizin-Berlin, Berlin, Germany. ${ }^{16}$ Centro Lucio Bini, Cagliari e Roma, Cagliari, Italy. ${ }^{17}$ Central Institute of Mental Health, Medical Faculty Mannheim, Heidelberg University, Mannheim, Baden-Württemberg, Germany. ${ }^{18}$ Montreal Neurological Institute, McGill University, Montreal, QC, Canada. ${ }^{19}$ Institute of Psychiatric Phenomics and Genomics, Munich, Germany. ${ }^{20}$ Department of Adult Psychiatry, Poznan University of Medical Sciences, Poznan, Poland. ${ }^{21}$ Department of Mental Health, Poznan University of Medical Sciences, Poznan, Poland. ${ }^{22}$ Department of Psychiatry, McGill University Health Centre, Montreal, Québec, Canada. ${ }^{23}$ Department of Psychiatry, Charles University, Prague, Czech Republic. ${ }^{24}$ Department of Psychiatric Nursing, Poznan University of Medical Sciences, Poznan, Poland. ${ }^{25} \mathrm{Harvard}$ Medical School and McLean Hospital, Boston, MA, USA
\end{abstract}

\section{Conflict of interest}

The authors declare that they have no conflict of interest.

\section{Publisher's note}

Springer Nature remains neutral with regard to jurisdictional claims in published maps and institutional affiliations.

Supplementary Information accompanies this paper at (https://doi.org/ 10.1038/s41398-020-01148-y).

Received: 14 February 2020 Revised: 20 October 2020 Accepted: 8 December 2020

Published online: 11 January 2021

\section{References}

1. Chesney, E., Goodwin, G. M. \& Fazel, S. Risks of all-cause and suicide mortality in mental disorders: a meta-review. World Psychiatry 13, 153-160 (2014).

2. Manchia, M. et al. Genetic risk of suicidal behavior in bipolar spectrum disorder: analysis of 737 pedigrees. Bipolar Disord. 15, 496-506 (2013).

3. Yatham, L. N. et al. Canadian Network for Mood and Anxiety Treatments (CANMAT) and International Society for Bipolar Disorders (ISBD) 2018 guidelines for the management of patients with bipolar disorder. Bipolar Disord. 20, 97-170 (2018).

4. Drancourt, N. et al. Duration of untreated bipolar disorder: missed opportunities on the long road to optimal treatment. Acta Psychiatr. Scand. 127, 136-144 (2013).

5. Nunes, A. et al. Prediction of lithium response using clinical data. Acta Psychiatr. Scand. 141, 131-141 (2020).

6. Grof, P. Responders to long-term lithium treatment. In Lithium in Neuropsychiatry: The Comprehensive Guide. (eds. Bauer, M., Grof, P. \& Muller-Oerlinghausen, B.) 157-178 (Informa Healthcare, UK, 2006).

7. Grof, P. et al. Is response to prophylactic lithium a familial trait? J. Clin. Psychiatry 63, 942-947 (2002).

8. Amare, A. T., et al. Association of polygenic score for major depression with response to lithium in patients with bipolar disorder. Mol. Psychiatry. http:// www.nature.com/articles/s41380-020-0689-5 (2020). In press

9. Amare, A. T. et al. Association of polygenic score for schizophrenia and HLA antigen and inflammation genes with response to lithium in bipolar affective disorder: a genome-wide association study. JAMA Psychiatry. 75, 65-74 (2018).

10. Hou, L. et al. Genetic variants associated with response to lithium treatment in bipolar disorder: a genome-wide association study. Lancet 387, 1085-1093 (2016).

11. Manchia, M. et al. Assessment of response to lithium maintenance treatment in bipolar disorder: a Consortium on Lithium Genetics (ConLiGen) Report.PLoS ONE 8, e65636 (2013).

12. Breiman, L. Random forests. Mach. Learn. 45, 5-32 (2001).

13. Pedregosa, F. et al. Scikit-learn: machine learning in Python. J. Mach. Learn. Res. 12, 2825-2830 (2012).

14. He, H. \& Garcia, E. Learning from imbalanced data sets. IEEE Trans. Knowl. Data Eng. 21, 1263-1264 (2010).

15. Mi, H. et al. Protocol update for large-scale genome and gene function analysis with the PANTHER classification system (v.14.0). Nat. Protoc. 14, 703-721 (2019).

16. Gershon, S., Chengappa, K. \& Malhi, G. Lithium specificity in bipolar illness: a classic agent for the classic disorder. Bipolar Disord. 11, 34-44 (2009).

17. Alda, M. The phenotypic spectra of bipolar disorder.Eur. Neuropsychopharmacol. 14, S94-S99 (2004).

18. Passmore, M. et al. Phenotypic spectra of bipolar disorder in responders to lithium versus lamotrigine. Bipolar Disord. 5, 110-114 (2003).

19. Kleindienst, N., Engel, R. \& Greil, W. Which clinical factors predict response to prophylactic lithium? A systematic review for bipolar disorders. Bipolar Disord. 7, 404-417 (2005).

20. Hui, T. et al. A systematic review and meta-analysis of clinical predictors of lithium response in bipolar disorder. Acta Psychiatr. Scand. 140, 94-115 (2019).

21. Backlund, L., Ehnvall, A., Hetta, J., Isacsson, G. \& AAgren, H. Identifying predictors for good lithium response - a retrospective analysis of 100 patients with bipolar disorder using a life-charting method. Eur. Psychiatry 24, 171-177 (2009).

22. Tondo, L., Hennen, J. \& Baldessarini, R. Rapid-cycling bipolar disorder: effects of long-term treatments. Acta Psychiatr. Scand. 108, 4-14 (2003).

23. Kleindienst, N. \& Greil, W. Differential efficacy of lithium and carbamazepine in the prophylaxis of bipolar disorder: results of the MAP study. Neuropsychobiology 42, 2-10 (2000).

24. Kendler, K. From many to one to many - the search for causes of psychiatric illness. JAMA Psychiatry 76, 1085-1091 (2019).

25. Cruceanu, C. et al. Rare susceptibility variants for bipolar disorder suggest a role for G protein-coupled receptors. Mol. Psychiatry 23, 2050-2056 (2018).

26. Bezchlibnyk, Y. \& Young, L. The neurobiology of bipolar disorder: focus on signal transduction pathways and the regulation of gene expression. Can. J. Psychiatry 47, 135-148 (2002).

27. Gonzalez-Maeso, J. \& Meana, J. Heterotrimeric G proteins: insights into the neurobiology of mood disorders. Curr. Neuropharmacol. 4, 127-138 (2006). 
28. Saxena, $A$. et al. Role of protein kinase $C$ in bipolar disorder: a review of the current literature. Mol. Neuropsychiatry 3, 108-124 (2017).

29. Vosahlikova, M. \& Svoboda, P. Lithium - therapeutic tool endowed with multiple beneficiary effects caused by multiple mechanisms. Acta Neurobiol. Exp. (Wars.) 76, 1-19 (2016).

30. Geoffroy, P. A. et al. Lithium response in bipolar disorders and core clock genes expression. World J. Biol. Psychiatry 19, 619-632 (2018).

31. McCarthy, M. J. et al. Chronotype and cellular circadian rhythms predict the clinical response to lithium maintenance treatment in patients with bipolar disorder. Neuropsychopharmacology 44, 620-628 (2019).

32. Hunsberger, J. G. et al. Novel integrative genomic tool for interrogating lithium response in bipolar disorder. Transl. Psychiatry 5, e504 (2015).

33. Reinbold, C. S. et al. Analysis of the influence of microRNAs in lithium response in bipolar disorder. Front. Psychiatry 9, 207 (2018).
34. Maloney, B. et al. Lithium alters expression of RNAs in a type-specific manner in differentiated human neuroblastoma neuronal cultures, including specific genes involved in Alzheimer's disease. Sci. Rep. 9, 18261 (2019).

35. Schnack, H. \& Khan, R. Detecting neuroimaging biomarkers for psychiatric disorders: sample size matters.Front. Psychiatry 7, 50 (2016).

36. Nunes, A. et al. Using structural MRI to identify bipolar disorders - 13 site machine learning study in 3020 individuals from the ENIGMA Bipolar Disorders Working Group.Mol. Psychiatry 25, 2130-2143 (2020).

37. Mertens, J. et al. Differential responses to lithium in hyperexcitable neurons from patients with bipolar disorder. Nature 527, 95-99 (2015).

38. Schulze, T. et al. The international consortium on lithium genetics (ConLiGen): an initiative by the NIMH and IGSLI to study the genetic basis of response to lithium treatment. Neuropsychobiology 62, 72-78 (2010). 